\title{
SCIDiC
}

International Journal of Forensic Science \& Pathology (IJFP)

ISSN: 2332-287X

\section{Spectrum Of Histopathological Lesions In Lungs At Medico Legal Autopsy: A Ten Years Study}

Research Article

\author{
Dr Manjula $\mathrm{K}^{1 *}$, Dr Srinivasa Reddy $\mathrm{P}^{2}$
}

${ }^{1}$ Professor, Sri Devaraj Urs Medical College, Sri DecarajUrs Academy of Higher Education and Research, Tamaka ,Kolar, Karnataka 563101.

${ }^{2}$ Professor and HOD Forensic medicine, Sri Devaraj Urs Medical College, Sri Devaraj Urs Academy of Higher Education and Research, Tamaka, Kolar-563101.

\section{Abstract}

Introduction: Various histopathological findings unrelated to the cause of death are noticed in routine histopathological examination of medico legal autopsies. Lungs are unique among all internal organs in that they are exposing themselves directly and constantly to the surrounding atmosphere and are important vital organ in human body. They are vulnerable for a wide range of inflammatory, infectious and neoplastic conditions. Despite recent advances in diagnostic technology, pulmonary diseases are sometimes nonspecific, pathological examination gives exact cause of death and helps to correlate clinical scenario. Organ specific pathological changes that are seen during autopsy give a clear picture of the cause of death and help to correlate with clinical features.

Materials And Methods: This retrospective study was done in the department of pathology, Sri Devaraj Urs Medical College on medico legal autopsies after taking ethical clearance from the institution. In all the cases available clinical details (age, sex, clinical diagnosis) was collected from medical records. Later gross details of lung specimens were collected. Gross and microscopic details were studied in details and analysed

Results: Total of about 154 lung specimens were studied. Male to female ratio was 1.8:1. Commonest age group was 20-29 $(22.72 \%) 3$ rd decade. Most common cause of death was Road traffic accident (19.48\%). Pulmonary oedema was the most common histopathological finding.

Conclusion: Autopsy is the gold standard to confirm the cause of death. Male to female ratio was 1.8:1. Commonest age group was 20-29 (22.72\%) 3rd decade. Most common cause of death was Road traffic accident (19.48\%). Pulmonary oedema was the most common histopathological finding in the medico legal autopsy of lung.

Keywords: Histopathology; Lungs; Autopsy.

\section{Introduction}

An autopsy is a medical practice that consists of a thorough examination done on a body after death and should be a gold stranded to ascertain the cause of death[1]. Medico legal autopsies are performed on the instructions of the legal authority in circumstances related to suspicious, sudden, obscure, unnatural or criminal deaths[2,3]. Various histopathological findings unrelated to the cause of death are noticed in routine histopathological examination of medico legal autopsies[4,5].

Lungs are unique among all internal organs in that they are exposing themselves directly and constantly to the surrounding atmosphere and are important vital organ in human body [6]. They are vulnerable for a wide range of inflammatory, infectious and neoplastic conditions $[6,7]$. Despite recent advances in diagnostic technology, pulmonary diseases are sometimes nonspecific, pathological examination gives exact cause of death and helps to correlate clinical scenario[8,9]. Organ specific pathological changes that are seen during autopsy give a clear picture of the cause of death and help to correlate with clinical features [6,9].

\section{Materials And Methods}

This retrospective study was done in the department of pathology, Sri DevarajUrs Medical College on medico legal autopsies after taking ethical clearance from the institution. In all the cases available clinical details (age, sex, clinical diagnosis) was collected

*Corresponding Author:

Dr. Manjula K

Professor, Dept. of Pathology, Sri Devaraj Urs Medical College, Sri DecarajUrs Academy of Higher Education and Research, Tamaka , Kolar, Karnataka 563101.

Tel : 8951374212

E-mail : Gkpmanju966@rediffmail.com

Received: June 01, 2021

Accepted: June 29, 2021

Published: July 19, 2021

Citation: Manjula K, Srinivasa Reddy P. Spectrum of histopathological lesions in lungs at medico legal autopsy: A ten years study. Int J Forensic Sci Pathol. 2021;8(2):437-440. doi: http://dx.doi.org/10.19070/2332-287X-2100092

Copyright: Manjula $\mathbf{K}^{\odot}$ 2021. This is an open-access article distributed under the terms of the Creative Commons Attribution License, which permits unrestricted use, distribution and reproduction in any medium, provided the original author and source are credited. 
from medical records. Later gross details of lung specimens were collected. Details regarding type of specimen, size and weight, characteristic of lesions (Shape, size, consistency, colour, and distribution), Extent and distribution of abnormalities noted in the lungs. Also noted changes seen in pleura(Color, thickness, plaques, nodules). Later bits were taken for microscopic study. At least one block from each lobe to include normal, most abnormal, and intermediate areas. At least one block of the large airways (if present). At least one block from pleura and Nodes was taken. In cases of neoplastic lesions careful description of the appearance and extent of the tumour was done with particular reference to invasion of adjacent structures. After routine processing, staining was done with Haematoxyline and Eosin( H\&E) All the histopathological sections were reviewed from the department archives ( microscopic findings), findings were noted. Special staining was used wherever necessary (Periodic Acid Schiff). Microscopic findings were analysed in details and tabulated.

\section{Inclusion Criteria}

All medico legal autopsies of cases aged more than 10 years.

\section{Exclusion Criteria}

Autolysed specimen

\section{Results}

Total of about 154 lung specimens were studied. The following observations were made; male to female ratio was 1.8:1. Commonest age group was 20-29 (22.72\%) 3rd decade. Table 1 shows age group distributions of lung autopsies

Most common cause of death was Road traffic accident (19.48\%) followed by sudden death $(18.18 \%)$, poisoning $(12.98 \%)$, hanging $(11.03 \%)$, found dead $(11.03 \%)$. Table 2 shows frequency of cause of death in medico legal lung autopsies

The most common gross finding was congestion of lungs accounting to $25.32 \%$, followed by lung consolidation in $22.32 \%$. Other gross findings were shown in table 3 .

The most common histopathological findings was pulmonary oedema $(38.31 \%)$ followed by normal lung $(20.77 \%)$, lobar pneumonia $(9.09 \%)$ and interstitial congestion $(7.79 \%)$. Tuberculosis was seen in 4cases, two in younger age 19 years and 30 years. Grossly grey white nodules were seen. Two cases were seen in older age group. One case was cavitatory lesion in an 86year male, another case in 65 year female patient presented with large area of consolidation. Autopsy of 67 years male lung revealed adenocarcinoma of lung presented grossly as a grey white nodule at the periphery with central necrosis. Cause of death was sudden death. Table 4 shows spectrum of histopathological lesions seen in lungs at medico legal autopsy.

\section{Discussion}

Autopsy has been regarded as a gold standard tool for the assessment of clinical diagnosis. Lung examination is the most important part of both the medico legal and clinical autopsy. Pathological examination of autopsy lungs gives valuable information on various stages of diseases and may reveal diagnosis which may not be suspected clinically $[1,2,3]$.

In the present study 154 cases of lung autopsy were studied. Male to female ratio was $1.8: 1$, similar to study done by $[1,3,9]$ commonest age group was $20-29(22.72 \%)$ 3rd decade, followed by 4 th decade. Similar observations were noted by $[4,10]$. In a study done by[6,3]commonest age group was 4th decade. In our study, Most common cause of death was Road traffic accident (19.48\%) followed by sudden death (18.18\%). In a study done by [1] most common cause of death was death due to unknown cause and sudden death.

In present study, pulmonary oedema was the most common histopathological finding accounting to $38.31 \%$ seen more in males. Similar observations were made by other authors in their studies $[2,6,11]$.

Histopathological examination of lung was unremarkable in $20.77 \%$ of autopsies. In other similar studies percentage of normal lungs varied from $2 \%$ to $26 \%$. $[1,2,4,5]$. Pneumonia (lobar, interstial and patchy) constituted $14.84 \%$. In other similar studies pneumonia accounted to $7.99 \%-26.31 \%$ [1-8]. Emphysema in our study accounted to $3.24 \%$. In similar studies done by other authors, the percentage of incidence varies from $5.5 \%$ to $77.5 \%$. This wide variation in incidence may be due to difference in geographic locality, different occupations, patient's habits and exposure to different air pollutions.

CVC lung was seen in 4 cases accounting to $2.59 \%$ which is less compare to other similar studies. Patel CB reported 26.44\% [6], Kaur B reported 19\%[3] and Kandy NC reported $18.42 \%$. Tuberculosis of lung was seen in 4 cases accounting to $2.59 \%$. The

Table 1: age group distribution of lung autopsies.

\begin{tabular}{|c|c|c|}
\hline Age & Number of cases & Percentage \\
\hline $10-19$ & 16 & 10.38 \\
\hline $20-29$ & 35 & 22.72 \\
\hline $30-39$ & 32 & 20.77 \\
\hline $40-49$ & 32 & 20.77 \\
\hline $50-59$ & 19 & 12.33 \\
\hline $60-69$ & 11 & 7.14 \\
\hline $70-79$ & 09 & 5.84 \\
\hline
\end{tabular}


Table 2 shows frequency of cause of death in medico legal lung autopsies.

\begin{tabular}{|c|c|c|}
\hline Cause of death & Number of cases & Percentage \\
\hline Road traffic accident & 30 & 19.48 \\
\hline Sudden death & 28 & 18.18 \\
\hline Poisoning & 20 & 12.98 \\
\hline Hanging & 17 & 11.03 \\
\hline Found dead & 17 & 11.03 \\
\hline Drowning & 09 & 5.84 \\
\hline Assault & 11 & 7.14 \\
\hline Snake bite & 10 & 6.49 \\
\hline Burns & 7 & 4.54 \\
\hline Bee sting & 5 & 3.24 \\
\hline Total & 154 & $100 \%$ \\
\hline
\end{tabular}

Table 3 shows various gross findings seen in lungs.

\begin{tabular}{|c|c|c|}
\hline Gross finding & Number of cases & Percentage \\
\hline Congestion & 39 & 25.32 \\
\hline Consolidation & 35 & 22.72 \\
\hline Firm & 23 & 14.93 \\
\hline Unremarkable & 16 & 10.38 \\
\hline Haemorrhage & 15 & 9.74 \\
\hline Petechial haemorrhage & 9 & 5.84 \\
\hline Fibrocavitary lesion & 6 & 3.8 \\
\hline Frothy exudates & 4 & 2.59 \\
\hline Collapse & 4 & 2.59 \\
\hline Grey white nodule & 3 & 1.94 \\
\hline Total & 154 & $100 \%$ \\
\hline
\end{tabular}

Table 4: shows spectrum of histopathological lesions seen in lungs at medico legal autopsy.

\begin{tabular}{|c|c|c|}
\hline Microscopic findings & Number of cases & Percentage \\
\hline Pulmonary edema & 59 & 38.31 \\
\hline Normal lung & 32 & 20.77 \\
\hline Lobar pneumonia & 14 & 9.09 \\
\hline Congestion & 12 & 7.79 \\
\hline Patchy pneumonia & 6 & 3.81 \\
\hline Emphysema & 5 & 3.24 \\
\hline CVC lung & 4 & 2.59 \\
\hline TB Lung & 4 & 2.59 \\
\hline Chronic bronchitis & 4 & 2.59 \\
\hline Interstitial pneumonia & 3 & 1.94 \\
\hline Granulomatous lesion & 2 & 1.29 \\
\hline Micro thrombi in lungs & 2 & 1.29 \\
\hline & & \\
\hline Alveolar haemorrhage & 2 & 1.29 \\
\hline Lung collapse & 2 & 1.29 \\
\hline Bronchi with vegetable matter & 2 & 1.29 \\
\hline Adenocarcinoma of lung & 1 & 0.64 \\
\hline Total & 154 & $100 \%$ \\
\hline
\end{tabular}


Table 5: shows correlation between cause of death and spectrum of histopathological lesions.

\begin{tabular}{|c|c|c|c|c|c|c|c|c|c|c|}
\hline Microscopic findings & $\begin{array}{c}\text { Road traffic } \\
\text { accident }\end{array}$ & \begin{tabular}{|c} 
Sudden \\
death
\end{tabular} & Poisoning & Hanging & $\begin{array}{c}\text { Found } \\
\text { dead }\end{array}$ & Drowning & Assault & $\begin{array}{c}\text { Snake } \\
\text { bite }\end{array}$ & Burns & $\begin{array}{r}\text { Bee } \\
\text { sting }\end{array}$ \\
\hline Pulmonary edema & 8 & 11 & 7 & 6 & 7 & 8 & & 5 & 6 & 1 \\
\hline Normal lung & 10 & 6 & & 5 & 3 & 2 & 2 & & & 4 \\
\hline Lobar pneumonia & 2 & 2 & 5 & & 2 & & 1 & 2 & 2 & \\
\hline Congestion & 4 & & 2 & & & 3 & & 2 & & 1 \\
\hline Patchy pneumonia & & 1 & 2 & 1 & 1 & & & & 1 & \\
\hline Emphysema & 1 & & & & 2 & 1 & & 1 & & \\
\hline CVC lung & & 1 & 1 & & 2 & & & 1 & & \\
\hline TB Lung & 1 & 1 & & & 2 & & & & & \\
\hline Chronic bronchitis & 1 & 1 & 1 & & 1 & & & & & \\
\hline Interstitial pneumonia & 1 & & 1 & & & 1 & & & & \\
\hline Granulomatous lesion & 1 & 1 & & & & & & & & \\
\hline Micro thrombi in lungs & 1 & & 1 & & & & & & & \\
\hline Alveolar haemorrhage & & & & & & 1 & & & & 1 \\
\hline Lung collapse & 1 & & & & & & & 1 & & \\
\hline Bronchi with vegetable matter & & & & & 1 & 1 & & & & \\
\hline Adenocarcinoma of lung & & 1 & & & & & & & & \\
\hline
\end{tabular}

Figure 1.

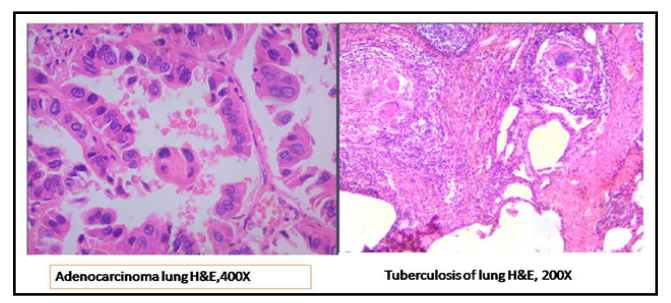

percentage of incidence in other studies varies widely. It is from $1.87 \%$ to $15.78 \%[1,7,8,10]$. In our study we had only one case of malignant primary lung lesion, adenocarcinoma in a 67 year male patient accounting to $0.64 \%$. it similar to study done by $[3,9]$ reported $2.08 \%$, [1] reported $0.41 \%$ and Tanaka et al reported $3.3 \%$ of cases of malignant primary lung lesion [12].

\section{Conclusion}

Autopsy is the gold standard to confirm the cause of death. Male to female ratio was 1.8:1. Commonest age group was 20-29 $(22.72 \%) 3$ rd decade. Most common cause of death was Road traffic accident (19.48\%). Pulmonary oedema was the most common histopathological finding in the medico legal autopsy of lung.

\section{References}

[1]. MangalK, Dhakar P, Yadav A, GuptaK, GandhiS. Magnitude of pulmonary diseases - incidentally diagnosed on autopsy - at largest hospital \& medical college of rajasthan. Int J Cur Res Rev.2016; 8(8):37-43.

[2]. Khare P, Gupta R, Ahuja M, Khare N, Agarwal S, Bansal D. Prevalence of
Lung Lesions at Autopsy: A Histopathological Study. J Clin Diagn Res. 2017 May;11(5):13-16. Pubmed PMID: 28658765.

[3]. Kaur B, Gupta RK, Singh H, AggarwalA, Kundal RK, AnandG, et al. Histopathological Pattern of Lungs on Post-Mortem Specimen-AStudyof100Cases. Ann. Int.Med.Den. Res.2017; 3(4):01-06.

[4]. RupaliRamraoKurawar RR, Vasaikar MS. Spectrum of Histomorphological Changes in Lungs at Autopsy: A 5 Year Study. APALM.2017; 4(1):107-111.

[5]. Selvambigai G, Amudhavalli S, Chakravarthi DCD, Ravi S. Histopathological study of lung in autopsy cases: a prospective study. Int J Res Med Sci. 2016; 4(11):4816-9.

[6]. Patel CB, Patel K, BhagatVM, Shah P. Pattern of histopathological lesions in lungautopsy. Int J Res Med Sci 2018; 6:279-83.

[7]. Jhaveri S, Dudhatra S. Study of Prevalence of Histopathological Lesions in Lung at Autopsy. NJMR 2017; 7(4):15153.

[8]. Nada Chettian Kandy NC, Pai MR, PhiliposeRT. Role of Histopathology on Autopsy Study: An Audit. SAS J. Med., 2015; 1(1):7-15.

[9]. Chauhan G, Agrawal M, ThakkarN, Parghi B. Spectrum of histopathological lesions in lung autopsy. J Res Med Den Sci 2015; 3(2):109-12.

[10]. Chakma P, Thounaojam MD, Devi M. Incidental lung pathologies in medicolegal autopsies: a study, IJFCM 2017; 4(3):189-194.

[11]. Selvam V, Selvi RT, Subramaniam PM, VijayanathV. Prevalence of common disease in lungs and liver: a histopathological study. Jour Pharm Biomed Sc 2011; 12(12):1-4.

[12]. Sanefuji H, Adachi H, Baba K, Oda S, Nakata H, Hayashi M et al. An autopsy case of miliary Tuberculosis\& review of autopsy cases in japan. J UOEH. 1984 Mar 1; 6(1):75-86. 